\title{
Portal Vein Thrombosis in Cirrhosis
}

\author{
Kaiser Raja, Mathew Jacob, Sonal Asthana \\ Department of Hepatology, Hepatobiliary Surgery \& Multiorgan Transplantation, Global Integrated Liver Care Program, BGS Global Hospitals, \\ Bangalore, India
}

\begin{abstract}
Portal vein thrombosis (PVT) is being increasingly recognized in patients with advanced cirrhosis and in those undergoing liver transplantation. Reduced flow in the portal vein is probably responsible for clotting in the spleno-porto-mesenteric venous system. There is also increasing evidence that hypercoagulability occurs in advanced liver disease and contributes to the risk of PVT. Ultrasound based studies have reported a prevalence of PVT in 10-25\% of cirrhotic patients without hepatocellular carcinoma. Partial thrombosis of the portal vein is more common and may not have pathophysiological consequences. However, there is high risk of progression of partial PVT to complete PVT that may cause exacerbation of portal hypertension and progression of liver insufficiency. It is thus, essential to accurately diagnose and stage PVT in patients waiting for transplantation and consider anticoagulation therapy. Therapy with low molecular weight heparin and vitamin $\mathrm{K}$ antagonists has been shown to achieve complete and partial recanalization in $33-45 \%$ and $15-35 \%$ of cases respectively. There are however, no guidelines to help determine the dose and therapeutic efficacy of anticoagulation in patients with cirrhosis. Anticoagulation therapy related bleeding is the most feared complication but it appears that the risk of variceal bleeding is more likely to be dependent on portal pressure rather than solely related to coagulation status. TIPS has also been reported to restore patency of the portal vein. Patients with complete PVT currently do not form an absolute contraindication for liver transplantation. Thrombectomy or thromboendovenectomy is possible in more than $75 \%$ of patients followed by anatomical end-to-end portal anastomosis. When patency of the portal vein and/or superior mesenteric vein is not achieved, only non-anatomical techniques (reno-portal anastomosis or cavo-portal hemitransposition) can be performed. These techniques, which do not fully reverse portal hypertension, are associated with higher morbidity and mortality risks in the short term. (J Clin Exp Hepatol 2013;4:320-331)
\end{abstract}

$\mathrm{N}$ on-tumoral portal vein thrombosis (PVT) is not an uncommon complication occurring during the course of liver cirrhosis, frequently in its advanced stages. Alteration of blood flow within the portal vein probably plays an important role in the development of PVT with a possible contribution from the altered coagulation state in end stage liver disease. There has been an increased recognition of PVT due to frequent diagnostic imaging in patients with cirrhosis and especially in those

Keywords: portal vein thrombosis, cirrhosis, anticoagulation, portocaval hemitransposition

Received: 27.9.2013; Accepted: 2.12.2013; Available online: 31.12 .2013

Address for correspondence: Kaiser Raja, Department of Hepatology, Global Integrated Liver Care Program, BGS Global Hospitals, \#67 Utarahalli Road, Kengeri, Bangalore 560 060, India.

E-mail: kaiser@transplantationliver.com

Abbreviations: PVT: portal vein thrombosis; MELD: model for end stage liver disease; PT: prothrombin time; INR: international normalized ratio; DVT: deep vein thrombosis; PE: pulmonary embolism; MTHFR: methylene-tetrahydrofolate reductase; TEG: thromboelastography; US: ultrasonography; LMWH: low molecular weight heparin; VKA: vitamin $\mathrm{K}$ antagonists; TIPS: transjugular intrahepatic portosystemic shunt; EVL: endoscopic variceal ligation; SMV: superior mesenteric vein; IVC: inferior vena cava

http://dx.doi.org/10.1016/j.jceh.2013.12.003 awaiting transplantation. Development of PVT in a cirrhotic patient is expected to lead to an increase in portal pressure and decreased blood flow to the liver, thus increasing the risk of gastrointestinal bleeding, worsening of liver function, and worsening of ascites. However, the exact impact of PVT on the natural history of cirrhosis remains unclear. There are asymptomatic cirrhotic patients in whom PVT is detected incidentally on imaging and it is unclear whether it would be beneficial to treat such patients. At present there is no consensus regarding the anticoagulant drug, duration of treatment and monitoring to patients with cirrhosis and PVT. Presence of PVT has relevance during liver transplantation, since restoring both portal and arterial blood flow to the allograft is a necessary condition for liver transplantation to be successful. In this setting, PVT may be a source of technical difficulties with a negative impact on outcome. Occasionally, it may represent a definitive contraindication for transplantation. This review examines issues concerning the incidence, predisposing factors, pathogenesis and management of nontumoral PVT in patients with cirrhosis and in candidates for liver transplantation. We also discuss surgical options in patients with extensive thrombosis undergoing liver transplantation. 
Table 1 Hemostatic abnormalities associated with liver disease.

\begin{tabular}{ll}
\hline Factors promoting bleeding & Factors promoting thrombosis \\
\hline $\begin{array}{l}\text { Decreased levels of the } \\
\text { following }\end{array}$ & Decreased levels of the following \\
\hline $\begin{array}{l}\text { Coagulation factors II, V, VII, } \\
\text { IX, X, XI }\end{array}$ & Protein C \\
\hline $\begin{array}{l}\alpha \text { 2-antiplasmin } \\
\text { Thrombin-activatable } \\
\text { fibrinolysis inhibitor }\end{array}$ & Protein S \\
\hline $\begin{array}{l}\text { Histidine-rich glycoprotein } \\
\text { Platelet abnormalities }\end{array}$ & Antithrombin $\alpha$-macroglobulin \\
\hline $\begin{array}{l}\text { Thrombocytopenia } \\
\text { Impaired platelet function }\end{array}$ & Heparin cofactor II \\
\hline $\begin{array}{l}\text { Impaired platelet-wall } \\
\text { interaction }\end{array}$ & Plasminogen \\
\hline $\begin{array}{l}\text { Enhanced platelet inhibition } \\
\text { by nitric oxide and } \\
\text { prostacyclin }\end{array}$ & Fon Willebrand factor \\
\hline \begin{tabular}{l} 
Fibrinogen abnormalities \\
\hline Qualitative
\end{tabular} \\
\hline $\begin{array}{l}\text { Quantitative } \\
\text { Increased level of plasma tissue-type plasminogen activator }\end{array}$ \\
\hline Nutritional deficiency (vitamin K, folate) \\
\hline
\end{tabular}

\section{PREVALENCE}

The prevalence of PVT in cirrhotic patients is quite variable and has been reported from $1 \%$ to $28 \%$ depending upon the modality used for diagnosis and whether PVT was detected radiologically or intra-operatively at the time of liver transplantation. ${ }^{1-11}$ Studies based on ultrasonography have reported a prevalence of $10 \%-28 \%$, in unselected cirrhotic patients excluding those with hepatocellular carcinoma. $^{8-11}$ Maruyama et al did a retrospective analysis of 150 patients with hepatitis B or C related cirrhosis followed up for a median period of 66 months and reported a cumulative overall incidence of PVT of $12.8 \%$ at 1 year, $18.6 \%$ at 3 years, $20 \%$ at 5 years, and $38.7 \%$ at $8-10$ years. Majority of these patients $(73.8 \%)$ had partial PVT. ${ }^{9}$ The prevalence of PVT most likely increases with the severity of cirrhosis. It has been reported to be quite low (1\%) in patients with well compensated cirrhosis $^{2}$ while it is reported to be $8 \%-26 \%$ in decompensated cirrhotics awaiting liver transplantation. ${ }^{3-7}$ The prevalence of PVT in candidates for transplantation seems to be similar to that found in cirrhotic patients who were not necessarily evaluated for transplantation but had similar disease severity. ${ }^{1,8,12}$ It has been observed that at the time of evaluation for transplant, the model for end stage liver disease (MELD) and Child-Pugh scores seems to be higher in patients with PVT than in those without. ${ }^{1,6}$ A significant number of patients may have unrecognized PVT. In recent series, up to $50 \%$ of patients with PVT were detected for the first time at the time of transplant surgery. 3,13 This may be due to either false negatives on imaging or to PVT occurring while on the waiting list. Even in patients undergoing systematic ultrasound at close intervals of three months, the rate of previously unrecognized thrombosis remains relatively high. ${ }^{13}$ In patients waiting for transplant, the 12 -month risk incidence of developing PVT has been reported in one study to be $7 \%{ }^{3}$

\section{PATHOPHYSIOLOGY}

The development of portal vein thrombosis in patients with end stage liver disease is a multifactorial process, resulting primarily from a reduction in portal blood flow and hypercoagulability. Traditionally, cirrhosis is considered as a hypocoagulable state and the degree of prolongation of prothrombin time (PT) and international normalized ratio (INR) has been taken as a marker of the severity of coagulopathy. The INR has been designed primarily to assess hypocoagulability in patients on vitamin $\mathrm{K}$ antagonists. In patients with liver disease it probably overestimates the bleeding risk. ${ }^{14}$ This might explain the paradox of the poor prediction of bleeding in cirrhotics even with marked prolonged of conventional coagulation tests. It appears that in the setting of hepatic synthetic impairment, both pro- and anticoagulant proteins are reduced to a similar degree and the net result in most cirrhotic patients is a compensated hemostatic balance with no tendency for bleeding or thrombosis. ${ }^{15}$ (Table 1). Various clinical as well as in-vitro studies have actually shown that some patients with cirrhosis may have a thrombotic potential. ${ }^{16}$

All procoagulant factors except factor VIII are reduced in hepatic insufficiency. By contrast, the levels of factor VIII/vWF are increased in cirrhosis. ${ }^{15,17}$ Since all of the components in the "extrinsic" pathway are produced by hepatocytes, the degree of prolongation of the PT has been used extensively as a measure liver synthetic function. However, even anticoagulants such as Proteins $\mathrm{C}$ and $\mathrm{S}$ as well as the levels of circulating protease inhibitors are reduced in hepatic insufficiency. ${ }^{18,19}$ The physiologic effects of a deficiency of anticoagulants is not reflected in the PT or APTT, which measure only the procoagulant side of the hemostatic pathway. In vivo it is always the balance between the procoagulant and anticoagulant factors that ultimately determines whether bleeding, thrombosis, or appropriate hemostasis will occur in a particular setting. The hemostatic balance in liver disease can be thought of not as intrinsically pro- or anticoagulant, but rather as a state in which there is a reduced ability to maintain this balance. In cirrhosis, there is a relatively balanced reduction in both pro- and 
anticoagulant proteins, and therefore the net result is that there is very little change in the ability of the system to generate hemostatic levels of thrombin under ordinary circumstances. $^{15,20}$ In patients with stable cirrhosis, thrombin generation was found to be normal in in-vitro studies, when the natural anticoagulant pathway was activated by the addition of thrombomodulin. ${ }^{20,21}$ However, when the coagulation system in cirrhotic patients is stressed, for example by infection the system has limited buffer capacity and gets tipped out of balance into either a state of hemorrhage or thrombosis. ${ }^{22}$ Patients with cirrhosis also suffer from defects of platelet function and number that can contribute to a bleeding tendency. ${ }^{23}$ However, the platelet defects get balanced by increased levels of factor VIIIa/vWF, which increases platelet adhesion. ${ }^{24}$

Three recent independent studies have been published specifically evaluating the prevalence of deep vein thrombosis (DVT) and pulmonary embolism (PE) in patients with cirrhosis. ${ }^{25-27}$ The incidence of DVT/PE ranged from $0.5 \%$ to $1.87 \%$ in these studies. Interestingly one fifth of cirrhotic patients were receiving antithrombotic prophylaxis with drugs or compression devices at the time they experienced the thrombotic event. ${ }^{26}$ Thus, even patients with cirrhosis and a prolonged PT receiving antithrombotic drugs can develop a venous thrombosis. Decreased hepatic synthetic function as reflected by albumin levels was associated with increased risk of DVT/PE, suggesting a greater tendency to thrombosis in advanced stages of cirrhosis. ${ }^{25,26}$ The incidence of PVT has also been reported to be higher in patients with more severe cirrhosis. ${ }^{12}$ Some interesting in-vitro evidence also supports these clinical findings. The ratio of the most powerful procoagulant (factor VIII) and anticoagulant (protein C) in plasma showed a balance strongly in favor of factor VIII, which indicates hypercoagulability. ${ }^{16}$ Factor VIII concentrations have been reported to be higher in patients with worse Child-Pugh grade. ${ }^{16}$ A recent study reported that high levels of factor VIII were independently associated with cirrhotic PVT, with odds ratio for thrombosis being 6.0 if factor VIII levels were above $129 \mathrm{IU} / \mathrm{dl}^{28}$

While in non-cirrhotic patients, PVT is related to prothrombotic states (myeloproliferative diseases and/or inherited coagulation disorders) in a significant percentage of patients, in cirrhotic patients portal hemodynamics also plays an important role in the development of PVT. ${ }^{29}$ Portal flow is reduced in cirrhotic livers due to increased intrahepatic vascular resistance and this phenomenon is further pronounced as liver disease progresses. ${ }^{30,31}$ This may be another reason to explain the increased prevalence of PVT in advanced cirrhotics as compared to early cirrhotics with well compensated disease. In one prospective study, reduced portal flow velocity below $15 \mathrm{~cm} / \mathrm{s}$ was the only independent variable that correlated with the risk of developing PVT at 1 year of follow-up. ${ }^{31}$ Patients who develop PVT have also been reported to have low portal flow volumes and high collateral vessel flow velocity and flow volume.

Another independent risk factor for PVT is the degree of thrombocytopenia. ${ }^{9,31}$ This seems paradoxical since low platelet count should logically predispose to bleeding. Possibly as cirrhosis and portal hypertension progresses, the resultant decrease in portal flow possibly outweighs a protective effect of low platelet count against thrombosis and the paradoxical finding of increased PVT with lower platelet counts may be thus related to decreased portal flows that occurs with progression of portal hypertension. ${ }^{32}$

Although limited, but some studies do report a thrombophilic genotype in up to $70 \%$ of patients with cirrhosis and PVT. Polymorphisms TT677 of methylenetetrahydrofolate reductase (MTHFR) and G20210A in the prothrombin gene were significantly more frequent in this group compared with that in controls. ${ }^{8,12,33}$ Apart from gene mutations, it is actually difficult to detect underlying prothrombotic condition in these patients due to the non-specific decrease in both coagulation factors and inhibitors. ${ }^{29}$

Among other factors that may increase the risk of PVT is endotoxemia. ${ }^{34}$ Endotoxemia is common with worsening liver disease when PVT is also more common. Development of endotoxemia may be a surrogate for severity of cirrhosis. ${ }^{35,36}$ The role of sclerotherapy and cyanoacrylate glue injection as a potential trigger factor for PVT is controversial but has been reported in literature. ${ }^{37-39}$ Such associations could be as a result of selection bias of patients with more severe portal hypertension.

\section{TESTS OF COAGULATION IN CIRRHOSIS}

The PT and APTT are used universally to test for coagulation in patients with cirrhosis even though these tests are known to be poor predictors of bleeding in this category of patients. These tests do not reflect the in vivo physiological state since they do not account for naturally occurring anticoagulants such as protein $\mathrm{C}$ and antithrombin, levels of which are also substantially reduced in cirrhotics. ${ }^{20,40} \mathrm{~A}$ test for coagulation that can assess thrombin generation in the presence of thrombomodulin is more appropriate in cirrhotics. The thrombin generation test is one such test in which physiological coagulation is activated with small amounts of tissue factor as a trigger and phospholipids that act as platelet substitutes. ${ }^{41,42}$ The test gives a measure of the amount of thrombin that a given plasma sample may generate under the specified experimental conditions and represents the balance between the pro- and anticoagulant proteins in plasma. The test mimics more closely with what occurs in vivo, but further clinical studies are needed to validate 
this test in cirrhotics, especially for patients whose condition is complicated by bacterial infections or endothelial dysfunction. Another technique known as Thromboelastography (TEG) provides continuous observation and tracing of all the hemostatic functions that lead to clot formation and dissolution. TEG was developed as a means to investigate primary hemostasis, coagulation, and fibrinolysis. In the past, the use of TEG was limited by poor standardization, poor reproducibility, and difficulty in interpretation. Recently, the concepts of thromboelastography have been coupled with software-based analysis and this is now available as a bedside tool. ${ }^{40}$ It is frequently used during such major surgical interventions such as liver transplantation and cardiovascular procedures. ${ }^{43}$ Further work is required to determine whether use of TEG to determine coagulation status will be clinically and therapeutically meaningful in patients with cirrhosis.

\section{CLINICAL MANIFESTATIONS AND NATURAL HISTORY}

PVT is usually an incidental radiological finding in patients with cirrhosis. This is especially true for partial PVT, where it is expected that portal flow is not dramatically affected by the development of thrombosis. In fact a significant proportion of patients (up to 50\%) with PVT at the time of transplant surgery were previously unrecognized. ${ }^{3,13}$ The impact of PVT on the outcome of cirrhosis is not been investigated systematically. Maruyama et al in their series of 150 patients showed that grade of ascites was significantly worse and spleen size was significantly larger in the PVT group. The mean serum albumin was significantly lower and Child-Pugh score was significantly worse at the time of detection of thrombosis compared with baseline values. However, the incidence of variceal bleeding and the post-treatment recurrence rate for varices were not significantly different between patients who developed PVT compared to patients who did not develop PVT. Survival also did not differ between the two groups. However, a multi-center study from Italy that included 450 patients with upper gastrointestinal bleeding showed that PVT was independently associated with a higher risk of failure to control acute variceal bleeding as well as increased the risk of re-bleeding. ${ }^{44}$ When PVT occurs in patients with advanced cirrhosis and severe portal hypertension, it is debatable whether PVT is a mere marker of advanced disease or an event actually contributing to further deterioration in liver function. ${ }^{1}$ In patients awaiting liver transplantation, PVT has not been shown to be an independent predictor of wait-list mortality. ${ }^{45}$ Ferreira et al have, however, reported that occurrence of PVT is associated with increased mortality even in patients with low Child-Pugh scores. ${ }^{46}$

Table 2 Trials of anticoagulation therapy for PVT in patients with cirrhosis.

\begin{tabular}{|c|c|c|c|c|c|}
\hline Reference & $\begin{array}{c}\text { No. of } \\
\text { patients }\end{array}$ & Therapy & Recanalization & $\begin{array}{c}\text { Bleeding } \\
\text { complications }\end{array}$ & Comments \\
\hline Senzolo et $\mathrm{al}^{52}$ & $\begin{array}{l}33 \text { (treated) } \\
21 \text { (controls) }\end{array}$ & LMWH & $\begin{array}{l}\text { Complete (12), } \\
\text { Partial (9) }\end{array}$ & 1 (non-variceal) & $\begin{array}{l}\text { Time interval between appearance of } \\
\text { thrombosis and anticoagulation }<6 \text { months } \\
\text { predicted chance of repermeation } \\
\text { Thrombus progression occurred in } 15 / 21 \text { non } \\
\text { anticoagulated patients and in } 5 / 33 \\
\text { anticoagulated patients }(P<0.001)\end{array}$ \\
\hline Delgado et $\mathrm{al}^{58}$ & 55 (treated) & $\begin{array}{l}\text { LMWH (26), VKA } \\
\text { (29) }\end{array}$ & $\begin{array}{l}\text { Complete (25), } \\
\text { Partial (8) }\end{array}$ & $\begin{array}{l}5 \text { (non-variceal), } \\
6 \text { (variceal) }\end{array}$ & $\begin{array}{l}\text { Early initiation of anticoagulation improved } \\
\text { recanalization } \\
\text { Re-thrombosis after stopping anticoagulation } \\
\text { occurred in } 38.5 \% \\
\text { Less incidence of hepatic decompensation in } \\
\text { those with recanalization } \\
\text { Platelet count }<50,000 / \mathrm{mm}^{3} \text { increased } \\
\text { bleeding risk }\end{array}$ \\
\hline Amitrano et $\mathrm{al}^{60}$ & 28 (treated) & LMWH (Enoxaparin) & $\begin{array}{l}\text { Complete (21), } \\
\text { Partial (2) }\end{array}$ & None & $\begin{array}{l}\text { Complete PV recanalization rates increased } \\
\text { from } 33.3 \% \text { to } 75 \% \text { when duration of therapy } \\
\text { with LMWH was increased from } 6 \text { to } 12 \\
\text { months }\end{array}$ \\
\hline Francoz et $\mathrm{al}^{3}$ & 19 (treated) & $\begin{array}{l}\text { LMWH followed by } \\
\text { VKA }\end{array}$ & Complete (8) & None & $\begin{array}{l}\text { Low platelet count was a risk factor for PVT } \\
\text { Patient with PVT not receiving anticoagulation } \\
\text { had progression of thrombosis in } 60 \% \\
\text { Post-transplant survival was worse in patients } \\
\text { who had complete PVT and did not achieve } \\
\text { recanalization }\end{array}$ \\
\hline
\end{tabular}




\section{DIAGNOSIS OF PVT IN CIRRHOSIS}

Careful screening for PVT is important in all patients with cirrhosis and in those being evaluated for liver transplantation. Repeated imaging at specified intervals, usually every 3 months, during the pre-transplant waiting period is also recommended in order to detect thrombosis that may develop during follow-up. ${ }^{47}$ Patients who develop unexplained worsening of liver functions or gastrointestinal bleeding despite adequate prophylaxis should also be evaluated for new onset PVT. Both Doppler ultrasonography and multiphasic computed tomography have high sensitivity and specificity for detection of PVT. ${ }^{4-50}$ Doppler US is highly accurate at detecting thrombosis involving the trunk of the portal vein and in intrahepatic branches. It also provides additional information regarding portal flow and its direction. CT is better for assessing the superior mesenteric vein, spontaneous portosystemic shunts, renal veins, and the inferior vena cava. While a CT is generally performed at the time of initial evaluation for liver transplant, Doppler ultrasound is good for follow-up imaging as it can be performed repetitively and does not have the risks of intravenous iodine contrast and radiation. The resolution and definition of magnetic resonance imaging is lower than CT but it is an acceptable alternative in patients with impaired renal function. $^{51}$

In patients with HCC, it is essential to radiologically distinguish tumor invasion of the main trunk or the branches of the portal vein as the cause for PVT versus bland thrombus in the portal vein. This has implications since the major vascular tumoral invasion is an absolute contraindication to transplant, while bland PVT in the presence of HCC needs to be approached similar to a non-HCC setting. ${ }^{48}$ Tumor related PVT is usually detected in portal vein branches adjacent to and in direct continuity of the tumor, and is often associated with a high alpha fetoprotein level. Segmental dilation of the portal vein with arterial phase enhancement of the thrombus is diagnostic of tumor invasion. ${ }^{49}$

\section{RATIONALE FOR ANTICOAGULANT TREATMENT OF PVT IN CIRRHOSIS}

Several studies have reported that spontaneous recanalization of the portal vein in the absence of any specific therapy is unusual. In the study by Francoz et al no patient achieved recanalization in the absence of anticoagulation, while $42 \%$ achieved recanalization while on anticoagulant therapy. ${ }^{3}$ Senzolo et al reported thrombus progression in $75 \%$ of patients who did not receive anticoagulant treatment, compared to only $15 \%$ of treated patients. ${ }^{52}$ However, Maruyama et al reported spontaneous improvement in $47.6 \%$, unchanged appearance in $45.2 \%$ and progression in only $7.2 \%$. There was no significant difference in the natu- ral course of thrombosis based on the degree of obstruction or the location of the thrombus. Recurrence of PVT after spontaneous resolution was observed in $21.4 \%{ }^{9}$ The rationale for treating PVT in patients with cirrhosis is the fact that it increases morbidity compared to matched cirrhotics without PVT. PVT has been reported to be independently associated with a higher risk of failure to control acute variceal bleeding as well as increased the risk of rebleeding. ${ }^{44}$ Occurrence of PVT has also been shown to increase mortality and this has been observed even in patients with lower Child-Pugh scores. ${ }^{46}$ Recanalization of PVT has also been shown to reduce esophageal variceal pressure and this could also contribute to an improvement in morbidity and mortality. ${ }^{53}$

The importance of treating a cirrhotic patient who is being considered for liver transplantation and has PVT is to achieve recanalization and thus achieve a physiological portal vein anastomoses and ensure portal flow to the graft. Transplanting in the presence of extensive portal vein thrombosis makes surgery more complex and is associated with higher morbidity and mortality. Presence of PVT has been shown to be a predictor of post-transplant mortality in a large UNOS database and in other studies. ${ }^{1,6,45,54-57}$

\section{ANTICOAGULANT THERAPY}

Reviewing the limited studies reporting use of anticoagulation for PVT in patients with cirrhosis, complete recanalization has been described in $33 \%-45 \%$ while partial PV recanalization is observed in $15 \%-35 \%$ of cases ${ }^{3,58,59}$ (Table 2). In a study by Senzolo et al prospectively enrolling 56 individuals ( 35 treated and 21 controls) complete recanalization was achieved in $36 \%$ and partial recanalization in $27 \%$ individuals, after a mean of 5.5 months therapy with low molecular weight heparin $(\mathrm{LMWH}) .{ }^{52}$ Time between diagnosis and anticoagulation $<6$ months was the most important factor positively associated with PV recanalization. In this study there was progression of PVT in $15 \%$ of individuals in the treatment group compared to $71.4 \%$ in the untreated group. ${ }^{52}$ In a study from Spain by Delgado et al involving 55 cirrhotic individuals with a mean MELD score of 12.8 and majority (75\%) having partial PVT, complete PV recanalization was achieved in $45 \%$ of cases after a median duration of therapy of 6.3 months with vitamin $\mathrm{K}$ antagonists (VKA) or LMWH. ${ }^{58}$ In this study also the only predictive factor for complete PV recanalization was early initiation of anticoagulation after diagnosis ( $<14$ days). It has been shown that prolonging the duration of anticoagulation therapy can improve rates of complete PV recanalization. Amitrano et al treated 28 patients with LMWH (Enoxaparin $200 \mathrm{U} / \mathrm{kg} / \mathrm{d}$ ). PVT was partial in $83 \%$ of the cases and $46 \%$ of individuals had Child B or C cirrhosis. At 6 months complete PV recanalization was achieved in $33 \%$ and partial PV recanalization was observed in 50\% of 
the patients. In individuals with partial response to therapy, complete PV recanalization was achieved in $86 \%$ with continuation of enoxaparin for six more months. ${ }^{60}$ In most studies, recanalization is uncommon in patients with complete thrombosis, but anticoagulation still prevented the extension of the thrombus. ${ }^{3,58-60}$

An important objective in the management of PVT in cirrhotic patients awaiting liver transplantation is to achieve recanalization so that conventional end-to-end portal vein anastomosis is surgically possible. Another objective is to prevent extension of the thrombus to the splenic and superior mesenteric vein, since these veins can also be used to restore portal flow to the graft in case the main PV is thrombosed. In the event that neither the portal vein nor the superior mesenteric vein can be used, non-anatomical techniques to restore portal flow are possible but these are associated with increased morbidity and mortality. Francoz et al compared 19 individuals with cirrhosis and PVT on the waiting list for liver transplantation who received anticoagulation therapy (VKA) with 10 individuals not receiving therapy. $42 \%$ of treated individuals achieved complete PV recanalization. None of the untreated patients had recanalization and in fact PVT progressed in $60 \%$ in the untreated group. Anticoagulation therapy did not increase blood loss during liver transplantation. $^{3}$

\section{CHOICE OF ANTICOAGULATION}

There are no clear recommendations for an optimal anticoagulation regimen for the treatment of PVT in patients with cirrhosis. Monitoring of anticoagulation regimen is complex in the cirrhotic patient and therefore choosing between different anticoagulants (LMWH vs. VKA) is a difficult decision. LMWH, while as effective as VKA, is less practical for patients, since it necessitates daily subcutaneous injections. LMWH does not affect INR values and therefore does not interfere with MELD or Child scoring. There is, however, limited information on the pharmacodynamic profile of LMWH in cirrhotic individuals. Cirrhotic individuals often have an increased volume of distribution because of fluid overload and this makes it difficult to determine the optimal dose of $\mathrm{LMWH}^{61}$ LMWH is eliminated by the kidneys and since many patients with cirrhosis have renal insufficiency, the half-life of LMWH is increased. ${ }^{61}$ Monitoring of anti-Xa activity to guide therapy is unreliable in cirrhosis. ${ }^{59,61,62}$ The primary problem with VKA is determination of adequate anticoagulation in patient with cirrhosis who already have altered coagulation and abnormal prothrombin time. Most studies have targeted an INR of $2-3 .{ }^{58,63}$ This appears more of an empirical figure rather than based on physiological principles. If the baseline INR is over 2 , it is difficult to determine if a given dose of VKA ensures adequate anticoagulation. It may also be difficult to determine the optimal INR target for dose adjustment. There is also a potential risk of further lowering of protein C levels with the use of VKA and this could theoretically increase the prothrombotic imbalance of individuals with cirrhosis. ${ }^{62,64}$ Newer thrombin inhibitors and inhibitors of activated factor $\mathrm{X}$ such as dabigatran and rivaroxaban offer the advantage of oral administration, the absence of laboratory monitoring, and an antithrombin independent mechanism of action. ${ }^{65}$ However there are no reports regarding their use in cirrhotic patients who could be at risk of excessive anticoagulation with the use of these drugs. In cirrhotic patients, it may be necessary to reverse anticoagulation during episodes of inadvertent bleeding or at the time of surgery. While the effect of VKA can be effectively reversed by fresh frozen plasma or prothrombin complex concentrate, there is no potent and rapidly acting antidote to reverse the effect of LMWH or the newer thrombin inhibitors.

\section{SELECTION OF PATIENTS FOR ANTICOAGULATION}

It is uncertain whether it is beneficial to anticoagulate asymptomatic cirrhotic patients who are detected to have PVT incidentally on imaging. ${ }^{29,63}$ The impact of PVT on the natural history of cirrhosis is still a matter of great debate and the clinical benefits of PV recanalization have been very well demonstrated. ${ }^{60}$ Despite this, there is evidence that cirrhotic individuals with PVT on the waiting list for liver transplantation should be treated with anticoagulation therapy. Complete or partial PV recanalization has been associated with a better 2-year survival rate after liver transplantation (82-83\%) compared to individuals with complete PVT $(50 \%){ }^{3}$ Post transplantation survival of patients transplanted at a low MELD $(<15)$ is lower if they have PVT. ${ }^{56}$ It has also been shown that there is a higher risk of recurrent PVT after transplant in individuals who have PVT at the time of transplantation. ${ }^{62}$ Other logical situations where anticoagulation is expected to be beneficial are cirrhotic patients with acute PVT with extension to the superior mesenteric vein. ${ }^{29,63}$ However these situations are quite rare. Cirrhotic patients with documented to have a well characterized prothrombotic disorder (viz. JAK2 mutation) obviously should be considered for anticoagulation. Patients with cavernomatous transformation of the portal vein have been excluded in most trials since such patients are not expected to benefit from anticoagulation.

\section{TIPS AND THROMBOLYSIS}

Use of Transjugular intrahepatic portosystemic shunt (TIPS) has also been reported to recanalize the portal vein and, subsequently, prevent re-thrombosis by restoring 
portal flow through the shunt. ${ }^{66-70}$ TIPS insertion and recanalization is associated with disrupture of the thrombus and mechanical thrombectomy. However, TIPS in such cases is expected to be technically challenging with a higher failure rate and should be attempted only in experienced centers. TIPS may also carry a higher complication rate since patients have advanced cirrhosis with post procedure mortality particularly high if the MELD score is more than $18 .^{71}$ Systemic or in-situ thrombolysis has been reported in cirrhotic patients with PVT. ${ }^{53}$ In non-cirrhotic patients with acute PVT, rates of recanalization have been dismal with attempted thrombolysis. There has been a high incidence of major bleeding also. ${ }^{29,72}$ There is no data to support this option in this setting.

\section{PROPHYLAXIS FOR PVT}

Enoxaparin has been showed to prevent PVT in patients with advanced cirrhosis awaiting liver transplantation. Villa et al followed 34 outpatients with cirrhosis (Child class B7-C10) with patent portal veins and without hepatocellular carcinoma who were treated with daily subcutaneous enoxaparin (4000 IU/day) for one year. ${ }^{73}$ At the end of one year, none of the patients in the enoxaparin group had developed PVT, compared with 6 of 36 (16.6\%) controls. Enoxaparin was stopped and patients were followed up for an additional year. At the end of the follow-up period of two years, none of the patients in the enoxaparin group and $27.7 \%$ of controls developed PVT. PVT did develop in three enoxaparin treated patients in the third year. Liver decompensation was less frequent among patients given enoxaparin (11.7\%) than controls (59.4\%). Survival was also reported to be better in the enoxaparin group. No relevant side effects or hemorrhagic events were reported. Results of this study need to be reproduced in a larger study before anticoagulation can be recommended as a prophylactic therapy.

\section{COMPLICATIONS OF ANTICOAGULATION AND PROPHYLAXIS OF VARICEAL BLEEDING}

The most feared complication of anticoagulant drug use in the setting of cirrhosis with altered coagulation is gastrointestinal bleeding that can be life threatening. The risk of variceal bleeding is highly dependent on portal pressure. ${ }^{74}$ It is now accepted that associated coagulopathy whether related to the underlying liver disease or anticoagulant use does not precipitate variceal bleeding, but can make the bleeding more severe. In published studies, the overall incidence of bleeding complications with anticoagulant use in cirrhotics has been reported to vary from $5 \%$ to $35 \%$. This has included bleeding from causes related to portal hypertension (varices or portal hypertensive gastropathy) or unrelated causes viz. post dental extrac-
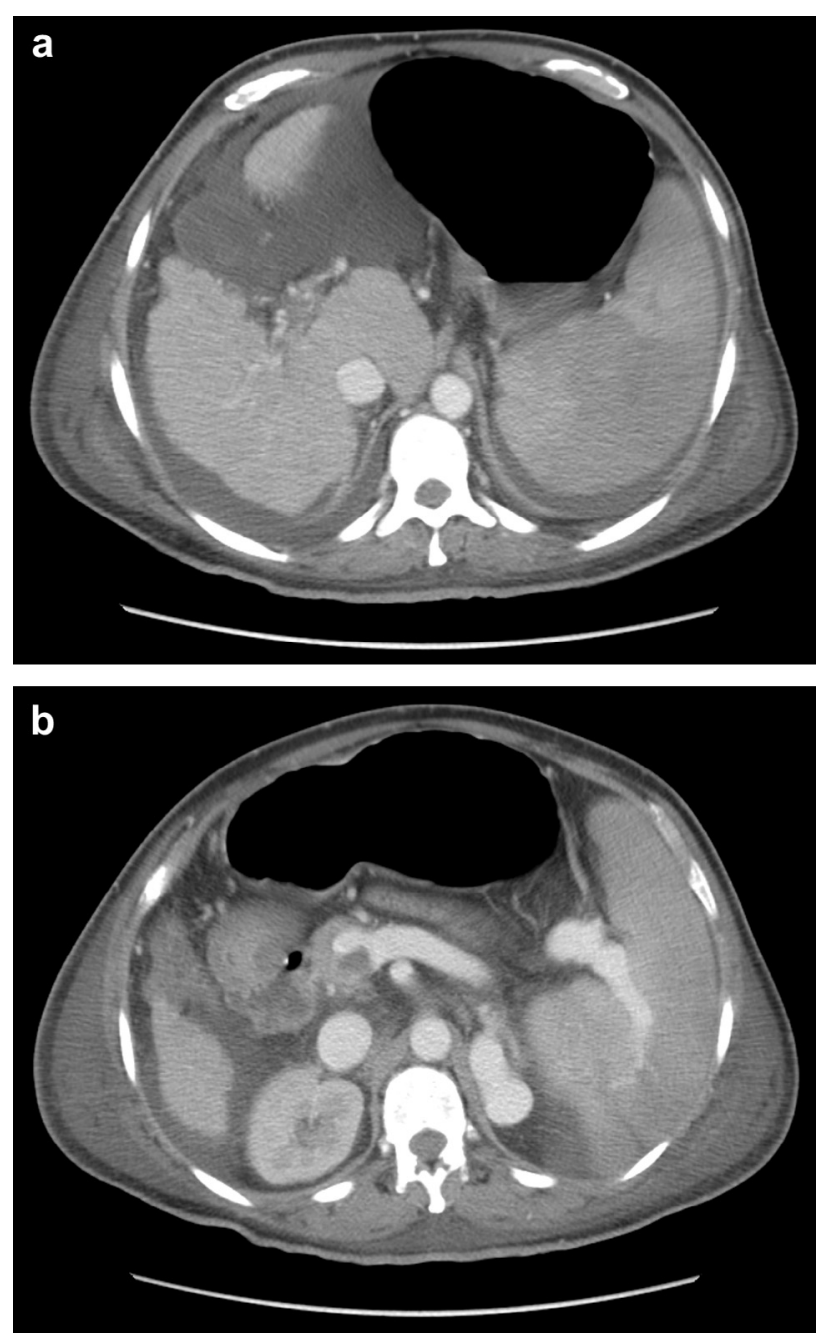

Figure 1 Contrast CT abdomen showing complete thrombosis of portal vein at the bifurcation with hilar collaterals (a) and non-occlusive thrombus in the main trunk of the portal vein extending to the confluence of the superior mesenteric vein (b).

tion, epistaxis, surgical wound hemorrhage, or vaginal bleeding., ${ }^{3,27,60,73}$ Obscure gastrointestinal bleeding (occult and overt) has also been reported. A platelet count $<50,000 / \mathrm{mm}^{3}$ and elevated creatinine have been associated with excess bleeding risk. ${ }^{58}$ Before initiating anticoagulant therapy in such patients the potential risks vs. benefits have to be addressed. The rate of variceal bleed has been generally low if cirrhotic individuals receive adequate prophylaxis. It is preferable to screen for varices before starting anticoagulation. However, in this particular situation, there is no consensus on whether nonselective beta-blockers, endoscopic variceal ligation (EVL), or combination therapy is better for variceal bleed prophylaxis. ${ }^{29,63,75,76}$ In case EVL is being performed (generally for large varices with red color signs or in patients with past history of variceal bleeding), it is recommended to delay initiation of anticoagulation therapy for atleast 2 weeks after the last EVL so as to allow post EVL ulcers to 

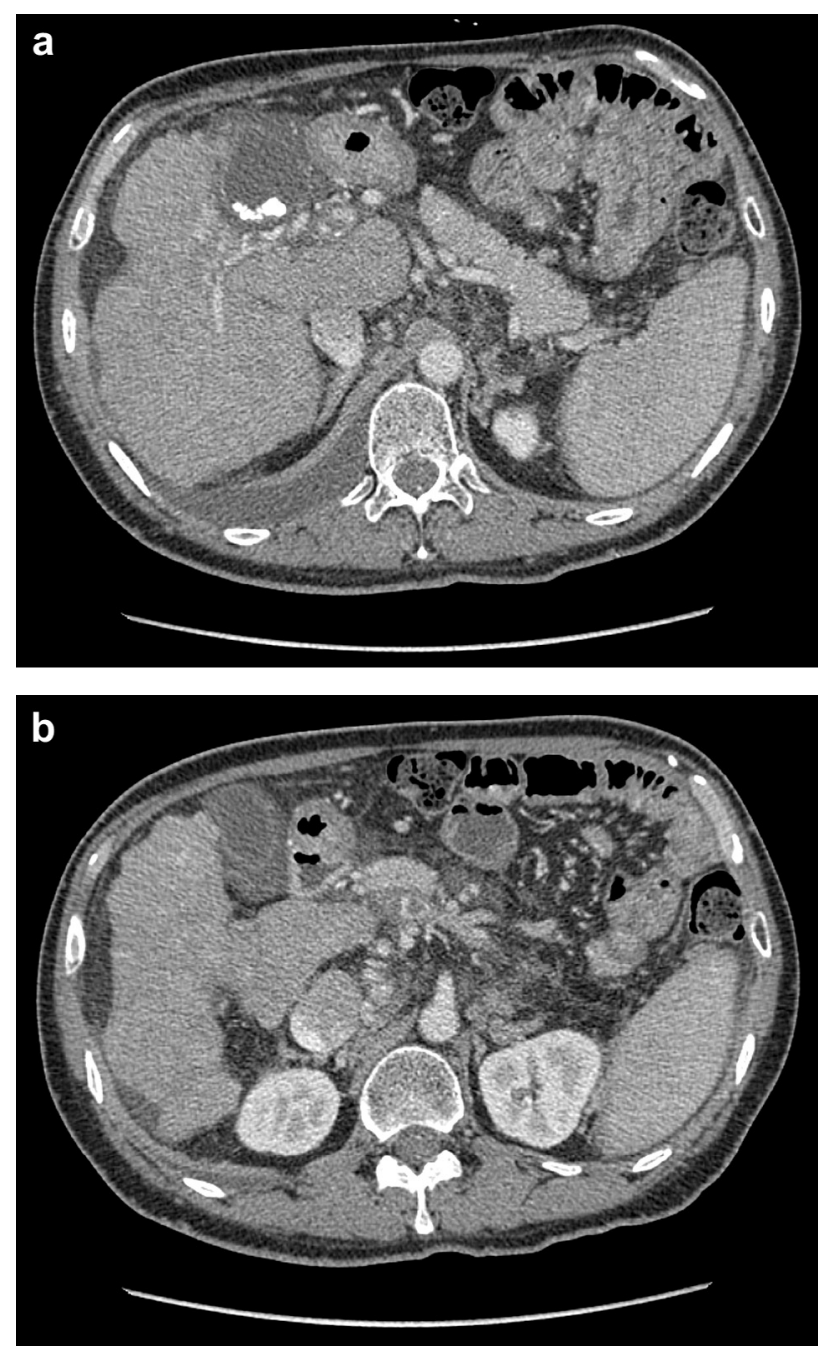

Figure 2 Contrast CT abdomen showing complete thrombosis of portal vein with multiple hilar collaterals (a) along with thrombosis of the superior mesenteric vein (b).

heal completely. ${ }^{52}$ In the published studies most patients have been on nonselective beta-blockers with or without EVL prior to initiating anticoagulation. It is important to determine the benefit of starting early anticoagulation in order to achieve rapid portal vein recanalization versus the potential risk of bleeding. Patients who have small to medium varices and have never bled in the past may be treated with nonselective beta-blockers instead of endoscopic variceal ligation and early anticoagulation initiated. $^{76}$

\section{SURGICAL TECHNIQUES FOR TRANSPLANTATION OF PATIENTS WITH CIRRHOSIS AND PVT}

PVT was considered as an absolute contraindication to liver transplantation in the initial stages of surgical evolution. ${ }^{77}$ The first successful surgery for complete PVT was
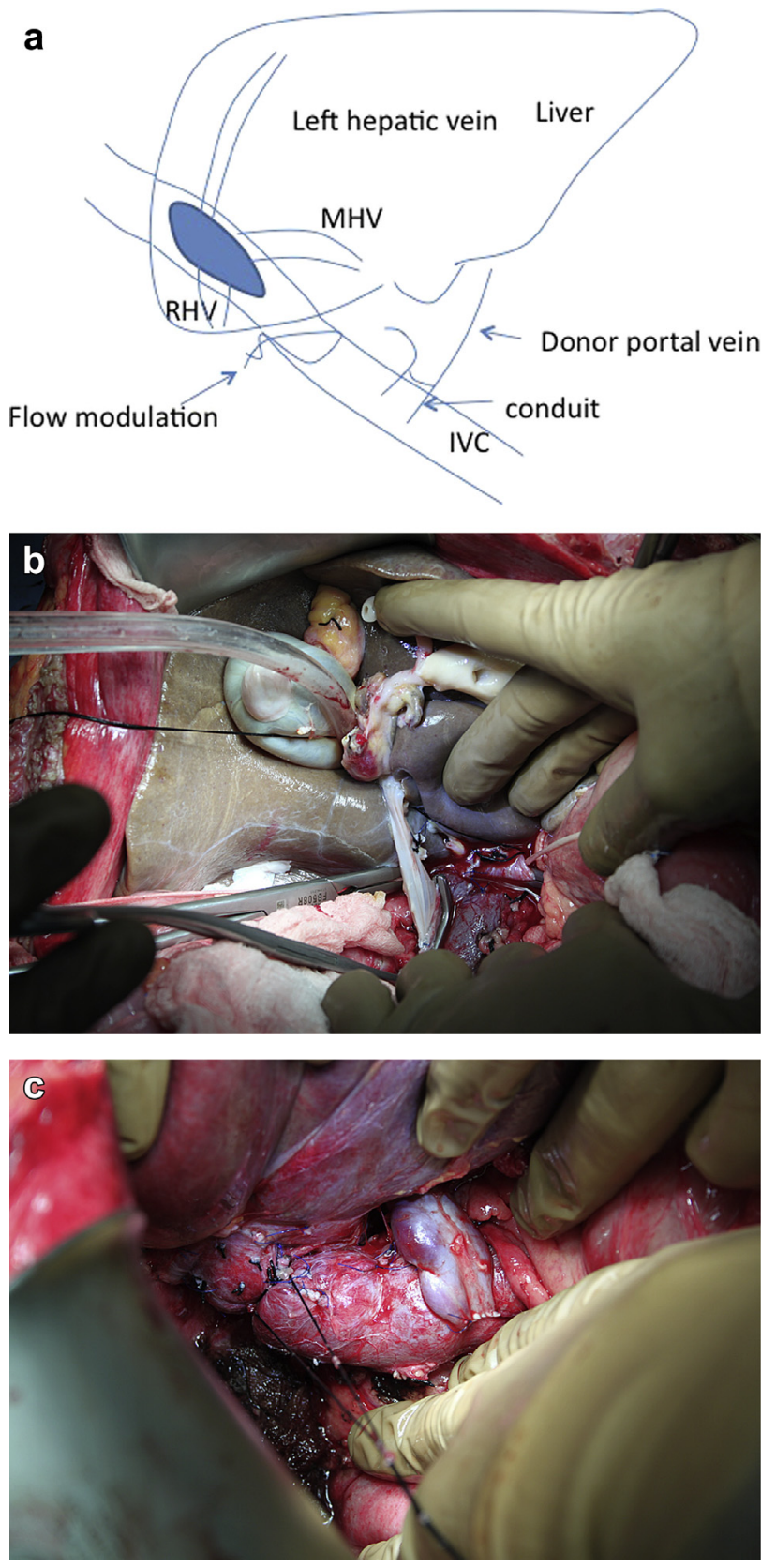

Figure 3 (a) Schematic diagram showing portocaval hemi-transposition with the IVC ligature to modulate blood flow. (b) Intra operative photograph showing the portocaval hemi-transposition prior to reperfusion of the graft. (c) Intra operative photograph showing ligature on the recipient IVC used to modulate blood flow in portocaval hemi-transposition.

reported by Shaw et al in $1985 .^{78}$ Innovations in surgical techniques have made it possible to overcome problems encountered due to PVT during transplantation. The preoperative condition and extensive collateral circulation of these patients add to the complexity of the involved surgical techniques, and remains a challenge for transplantation. An adequate portal inflow to the graft is essential for good liver function. There are several available surgical techniques for the reconstruction of the portal system to 
ensure restoration of adequate portal flow during LT. Preoperative assessment of the stage and grade of portal vein thrombosis is essential. Preoperative evaluation of these patients requires a dedicated tri-phasic spiral CT scan or an MR venogram. Yerdel et al have classified PVT into four grades, to aid surgical management. ${ }^{6}$

Grade 1: Minimally or partially thrombosed portal vein, where the thrombus is confined to $<50 \%$ of the lumen. Grade 2: More than 50\% occlusion of the portal vein, including total occlusions, with or without minimal extension into the SMV (Figure 1a,b).

Grade 3: Complete thrombosis of both the portal vein and the proximal superior mesenteric vein (SMV).

Grade 4: Complete thrombosis of the portal vein, proximal and distal SMV (Figure 2a,b).

Different approaches have been proposed to restore portal vein patency at the time transplantation. Approaches vary on the degree and the anatomical spread of the PVT. ${ }^{79}$

1. Portal vein thrombectomy and direct anastomosis of donor and recipient portal vein: Portal vein thrombectomy can be applied for Yerdel grade 1 and 2 PVT. A recent study suggested that $75 \%-90 \%$ of transplants performed in PVT could be managed by thrombectomy alone. ${ }^{79,80}$ This is achieved by freeing the recipient portal vein and gently developing the plane between the endothelium and the media of the vein by sharp dissection till patency is obtained. After completion of the thrombectomy, adequate flow in the recipient portal vein or SMV is confirmed by releasing the vascular clamp before proceeding with the anastomosis.

2. Use of jump graft: In cases of Yerdel grade 2 or grade 3 occlusions, an anastomosis may be required between the graft portal vein and the recipient SMV. This can be achieved using a section of donor iliac vein as a graft. The presence of a large collateral such as the coronary vein may provide an alternative portal inflow. Caution should be exercised as extra-anatomical vessels are extremely fragile and thin walled, as well as more prone to postoperative thrombosis.

3. Arterialization of the portal vein: Portal inflow can be achieved by anastomosing the graft portal vein to the recipient arterial inflow.

4. Portocaval hemitransposition (Figure 3a): An end-toside anastomosis of the graft portal vein is made to the suprarenal recipient inferior vena cava (IVC) (Figure $3 \mathrm{~b}$ ). Blood flow through the IVC is diverted toward the graft by incompletely ligating the retro-hepatic IVC with a silk suture to increase pressure and thereby flow in the portal vein (Figure 3c). A portal inflow pressure of $15-25 \mathrm{~cm} \mathrm{H}_{2} \mathrm{O}$ is considered adequate. Complete occlusion of the retro-hepatic IVC should be avoided in order to allow some of the IVC blood to flow back to the systemic venous pool. The disadvantage of classic portocaval hemitransposition is the persistence of portal hypertension and the ongoing risk of bleeding from gastroesophageal varices and portal hypertensive gastropathy, which may occur in up to $50 \%$ of such cases. ${ }^{80}$ Rodriguez-Castro, in a recent systematic review, reported that among 49 patients with portocaval hemitransposition, $20 \%$ had episodes of variceal bleeding, $58 \%$ had persistent ascites, and $26 \%$ presented with renal dysfunction after $\mathrm{LT}^{81}$ An alternative to portocaval hemitransposition is reno-portal transposition, where the recipient portal vein is anastomosed to the left renal vein. $^{82}$

5. Multivisceral transplantation: combined liver and small bowel transplantation is an infrequently used alternative in cases of bowel ischemia, and when no other portal inflow is available.

\section{OUTCOMES AFTER LIVER TRANSPLANTATION}

Most surgical series report higher technical difficulties, postoperative complications, inferior survival, and higher mortality, in cirrhotic patients with PVT undergoing liver transplantation. The higher morbidity and mortality is multifactorial and is related to a more complex surgical procedure, greater requirement of blood transfusions, increased risk of complications such as primary nonfunction or dysfunction, hepatic artery thrombosis, relaparotomy, postoperative pancreatitis, sepsis, and renal failure. ${ }^{4,83,84}$ Additionally, there is a high risk of rethrombosis of the portal vein $(9 \%-42 \%) .{ }^{81}$ The risk of rethrombosis has been reported to be higher in patients with higher grades of PVT, Child-Pugh class C cirrhosis, and in patients transplanted for alcoholic liver disease. Of a pooled total of 169 patients with partial PVT, 7 (4\%) developed re-thrombosis in contrast with 14 of 114 patients with complete PVT (12.3\%). ${ }^{81}$ Early anticoagulation with low molecular weight heparin appears to be protective unless contraindicated for surgical reasons, although powered evidence is lacking. Equally, there is no consensus on how long anticoagulation should be continued posttransplant. In the absence of prothrombotic states that may persist after transplantation, there is no evidence that pre-transplant portal vein thrombosis justifies long term anticoagulation post-transplantation, provided portal flow has been restored through conventional end-toend portal anastomosis. In our institution we continue anticoagulation for 6 months post transplantation for patients with PVT undergoing end-to-end portal anastomosis. The 30-day mortality in patients undergoing liver transplantation with or without PVT has been reported as $10.5 \%$ vs. $7.7 \%$, respectively $(P=0.01) .{ }^{81}$ The one-year mortality was also reported to be significantly higher in a systematic review according to the presence $(18.8 \%)$ or 
absence $(15.3 \%)$ of PVT $(P=0.001) \cdot{ }^{81}$ Mortality is related to the grade of preoperative PVT. The 30-day mortality has been reported to vary between $3.8 \%$ for grade 1 and 2 PVT, and going up to $27 \%$ for grade 4 PVT. ${ }^{85,86}$ Earlier studies had reported fairly dismal long term outcomes, especially after hemitransposition, although this situation has improved in the recent past. Preoperative PVT seems to influence early outcome more than delayed results, with the maximum drop-off in survival occurring in the first year. The medium-term results with or without PVT appear roughly comparable if the early mortality is excluded. ${ }^{79,87}$

\section{CONCLUSIONS}

PVT is a common problem in cirrhosis, mostly in individuals with advanced liver disease. PVT is an important prognostic factor of cirrhosis and also bears significance in individuals undergoing liver transplantation. Because many cases of PVT occur in patients who have decompensated Child's C cirrhosis, the symptoms of PVT, such as worsening of ascites or precipitation of an esophageal variceal bleeding event, may be attributed to worsening of cirrhosis without recognition of underlying PVT. Ultrasound may miss PVT unless Doppler imaging or contrast $\mathrm{CT}$ is done. PVT in cirrhosis is possibly due to low flow state in the portal vein and altered coagulation status. The coagulopathy of liver disease is extraordinarily complex and unpredictable, especially with the use of conventional tests such as prothrombin time and international normalized ratio. Despite the widespread belief that cirrhosis is a hypocoagulable state, patients with cirrhosis may develop venous thrombosis. The clinical impact of PVT on liver function is still a matter of great debate in the literature. PVT is a predictive factor for mortality, independent of MELD score, in individuals with cirrhosis. Since PVT by itself increases portal hypertension, it increases the risk of variceal bleeding and has been described to be an independent risk factor for the inability to control variceal bleeding. Although PVT is no longer an absolute contraindication for liver transplantation, patients with extensive thrombosis extending to the porto-mesenteric confluence are surgical challenges. Such patients require non-anatomical means of reconstruction of portal flow (cavo-portal or reno-portal), where the morbidity and short term mortality may be higher.

Anticoagulation therapy for PVT in cirrhotic individuals is associated with complete recanalization rates between $33 \%$ and $45 \%$ after 6 months. Prolonged anticoagulation could be associated with higher complete recanalization rates, lower rates of thrombosis extension, and lower rates of thrombosis recurrence after discontinuation of anticoagulation. Success rates for anticoagulation are higher for partial PVT compared to complete PVT. Both LMWH and VKA have been used for anticoagulation. It is unclear which is a better and safer option. It would probably be safer to use LMWH in cirrhotic individuals with abnormal INR. Bleeding complications secondary to portal hypertension in cirrhotic individuals undergoing anticoagulation for PVT seem to be low but prophylaxis for variceal bleeding probably needs to be administered to all patients. There is no definite recommendation in this setting but as in most other patient with cirrhosis and portal hypertension, EVL, nonselective beta-blockers or combination therapy has been used for prophylaxis. TIPS has also been reported as a salvage measure in PVT. It has a mechanical thrombolytic action and restores hepatopetal portal flow. Although, technical success rates in such situation may be as low as $70 \%$, recanalization rates are high. Primary prophylaxis of PVT has been attempted with low dose LMWH in a cohort of moderately severe cirrhotic individuals and has been reported to be highly effective in preventing PVT, reducing the risk of hepatic decompensation and reducing mortality. Future studies should help in understanding the exact clinical implications of PVT in patients with differing severity of cirrhosis. The correlation of portal flow dynamics as assessed on Doppler with the risk of PVT needs to be further studied. Exploration and validation of coagulation tests that reflect the exact in vivo physiological state of coagulation in patients with cirrhosis (such as thrombin generation test and thromboelastogram) and introduction of these tests for routine use will help identify subsets of patients with a thrombogenic profile as well as help in optimizing monitoring of anticoagulation. Identification of such patients at high risk for PVT and targeting them with anticoagulants as a primary prophylaxis may then be justifiable.

\section{CONFLICTS OF INTEREST}

All authors have none to declare.

\section{REFERENCES}

1. Englesbe MJ, Kubus J, Muhammad W, et al. Portal vein thrombosis and survival in patients with cirrhosis. Liver Transpl. 2010;16:8390.

2. Okuda K, Ohnishi K, Kimura K, et al. Incidence of portal vein thrombosis in liver cirrhosis. An angiographic study in 708 patients. Gastroenterology. 1985;89:279-286.

3. Francoz C, Belghiti J, Vilgrain V, et al. Splanchnic vein thrombosis in candidates for liver transplantation: usefulness of screening and anticoagulation. Gut. 2005;54:691-697.

4. Llado L, Fabregat J, Castellote J, et al. Management of portal vein thrombosis in liver transplantation: influence on morbidity and mortality. Clin Transplant. 2007;21:716-721.

5. Manzanet G, Sanjuan F, Orbis P, et al. Liver transplantation in patients with portal vein thrombosis. Liver Transpl. 2001;7:125-131.

6. Yerdel MA, Gunson B, Mirza D, et al. Portal vein thrombosis in adults undergoing liver transplantation: risk factors, screening, management, and outcome. Transplantation. 2000;69:18731881.

7. Gayowski TJ, Marino IR, Doyle HR, et al. A high incidence of native portal vein thrombosis in veterans undergoing liver transplantation. J Surg Res. 1996;60:333-338. 
8. Amitrano L, Brancaccio V, Guardascione MA, et al. Inherited coagulation disorders in cirrhotic patients with portal vein thrombosis. Hepatology. 2000;31:345-348.

9. Maruyama H, Okugawa H, Takahashi M, Yokosuka O. De novo portal vein thrombosis in virus-related cirrhosis: predictive factors and long-term outcomes. Am J Gastroenterol. 2013;108:568-574.

10. Fimognari FL, De Santis A, Piccheri C, et al. Evaluation of D-dimer and factor VIII in cirrhotic patients with asymptomatic portal venous thrombosis. J Lab Clin Med. 2005;146:238-243.

11. Gaiani S, Bolondi L, Li Bassi S, Zironi G, Siringo S, Barbara L. Prevalence of spontaneous hepatofugal portal flow in liver cirrhosis. Clinical and endoscopic correlation in 228 patients. Gastroenterology. 1991;100:160-167.

12. Amitrano L, Guardascione MA, Brancaccio V, et al. Risk factors and clinical presentation of portal vein thrombosis in patients with liver cirrhosis. J Hepatol. 2004;40:736-741.

13. Dumortier J, Czyglik O, Poncet G, et al. Eversion thrombectomy for portal vein thrombosis during liver transplantation. Am J Transplant. 2002;2:934-938.

14. Arjal R, Trotter JF. International normalized ratio of prothrombin time in the model for end-stage liver disease score: an unreliable measure. Clin Liver Dis. 2009;13:67-71.

15. Monroe DM, Hoffman M. The coagulation cascade in cirrhosis. Clin Liver Dis. 2009;13:1-9.

16. Tripodi A, Primignani M, Chantarangkul V, et al. An imbalance of pro- vs anti-coagulation factors in plasma from patients with cirrhosis. Gastroenterology. 2009;137:2105-2111.

17. Jennings I, Calne RY, Baglin TP. Predictive value of von Willebrand factor to ristocetin cofactor ratio and thrombin-antithrombin complex levels for hepatic vessel thrombosis and graft rejection after liver transplantation. Transplantation. 1994;57:1046-1051.

18. De Caterina M, Tarantino G, Farina C, et al. Haemostasis unbalance in Pugh scored liver cirrhosis: characteristic changes of plasma levels of protein C versus protein S. Haemostasis. 1993;23:229-235.

19. Raya-Sanchez JM, Gonzalez-Reimers E, Rodriguez-Martin JM, et al. Coagulation inhibitors in alcoholic liver cirrhosis. Alcohol. 1998;15:19-23.

20. Tripodi A, Salerno F, Chantarangkul V, et al. Evidence of normal thrombin generation in cirrhosis despite abnormal conventional coagulation tests. Hepatology. 2005;41:553-558.

21. Oliver JA, Monroe DM, Church FC, et al. Activated protein C cleaves factor Va more efficiently on endothelium than on platelet surfaces. Blood. 2002;100:539-546.

22. Violi F, Ferro D, Basili S, et al. Association between low-grade disseminated intravascular coagulation and endotoxemia in patients with liver cirrhosis. Gastroenterology. 1995;109:531-539.

23. Tripodi A, Primignani M, Chantarangkul V, et al. Thrombin generation in patients with cirrhosis: the role of platelets. Hepatology. 2006;44:440-445.

24. Lisman T, Bongers TN, Adelmeijer J, et al. Elevated levels of von Willebrand factor in cirrhosis support platelet adhesion despite reduced functional capacity. Hepatology. 2006;44:53-61.

25. Gulley D, Teal E, Suvannasankha A, Chalasani N, Liangpunsakul S. Deep vein thrombosis and pulmonary embolism in cirrhosis patients. Dig Dis Sci. 2008;53:3012-3017.

26. Northup PG, McMahon MM, Ruhl AP, et al. Coagulopathy does not fully protect hospitalized cirrhosis patients from peripheral venous thromboembolism. Am J Gastroenterol. 2006:101:1524-1528.

27. Garcia-Fuster MJ, Abdilla N, Fabia MJ, Fernandez C, Oliver V, Forner MJ. Venous thromboembolism and liver cirrhosis. Rev Esp Enferm Dig. 2008;100:259-262.

28. Martinelli I, Primignani M, Aghemo A, et al. High levels of factor VIII and risk of extra-hepatic portal vein obstruction. J Hepatol. 2009;50:916-922.
29. DeLeve LD, Valla DC, Garcia-Tsao G. Vascular disorders of the liver. Hepatology. 2009;49:1729-1764.

30. Shah V. Molecular mechanisms of increased intrahepatic resistance in portal hypertension. J Clin Gastroenterol. 2007;41:S259-S261.

31. Zocco MA, Di Stasio E, De Cristofaro R, et al. Thrombotic risk factors in patients with liver cirrhosis: correlation with MELD scoring system and portal vein thrombosis development. J Hepatol. 2009;51:682-689.

32. Lisman T, Porte RJ. The role of platelets in liver inflammation and regeneration. Semin Thromb Hemost. 2010;36:170-174.

33. Mangia A, Villani MR, Cappucci G, et al. Causes of portal venous thrombosis in cirrhotic patients: the role of genetic and acquired factors. Eur J Gastroenterol Hepatol. 2005;17:745-751.

34. Violi F, Ferro D, Basili S, et al. Ongoing prothrombotic state in the portal circulation of cirrhotic patients. Thromb Haemost. 1997;77:44-47.

35. Lin RS, Lee FY, Lee SD, et al. Endotoxemia in patients with chronic liver diseases: relationship to severity of liver diseases, presence of esophageal varices, and hyperdynamic circulation. J Hepatol. 1995;22:165-172.

36. Lumsden $\mathrm{AB}$, Henderson JM, Kutner $\mathrm{MH}$. Endotoxin levels measured by a chromogenic assay in portal, hepatic and peripheral venous blood in patients with cirrhosis. Hepatology. 1988;8:232236.

37. Amitrano L, Brancaccio V, Guardascione MA, et al. Portal vein thrombosis after variceal endoscopic sclerotherapy in cirrhotic patients: role of genetic thrombophilia. Endoscopy. 2002;34:535538.

38. Politoske D, Ralls P, Korula J. Portal vein thrombosis following endoscopic variceal sclerotherapy. Prospective controlled comparison in patients with cirrhosis. Dig Dis Sci. 1996;41:185-190.

39. Shim CS, Cho YD, Kim JO, et al. A case of portal and splenic vein thrombosis after histoacryl injection therapy in gastric varices. Endoscopy. 1996;28:461.

40. Tripodi A. Tests of coagulation in liver disease. Clin Liver Dis. 2009;13:55-61.

41. Hemker HC, Giesen P, Al Dieri R, et al. Calibrated automated thrombin generation measurement in clotting plasma. Pathophysiol Haemost Thromb. 2003;33:4-15.

42. Chantarangkul V, Clerici M, Bressi A, et al. Thrombin generation assessed as endogenous thrombin potential (ETP) in patients with hypo- or hyper-coagulability. Effects of phospholipids, tissue factor and residual platelets on the measurement performed in plateletpoor and platelet-rich plasma. Haematologica. 2003;88:547-554.

43. Koh MB, Hunt BJ. The management of perioperative bleeding. Blood Rev. 2003;17:179-185.

44. D'Amico G, De FR. Upper digestive bleeding in cirrhosis. Posttherapeutic outcome and prognostic indicators. Hepatology. 2003;38:599-612.

45. Englesbe MJ, Schaubel DE, Cai S, Guidinger MK, Merion RM. Portal vein thrombosis and liver transplant survival benefit. Liver Transpl. 2010;16:999-1005.

46. Ferreira CN, Rodrigues T, Alexandrino P, Ramalho F, Velosa JF. Portal vein thrombosis in cirrhotic patients is associated with advanced liver disease and predicts poor long-term prognosis. Hepatology. 2010;52:1072A.

47. Francoz C, Valla D, Francois D. Portal vein thrombosis, cirrhosis, and liver transplantation. J Hepatol. 2012;57:203-212.

48. Piscaglia F, Gianstefani A, Ravaioli M, et al. Criteria for diagnosing benign portal vein thrombosis in the assessment of patients with cirrhosis and hepatocellular carcinoma for liver transplantation. Liver Transpl. 2010;16:658-667.

49. Tublin ME, Dodd GD, Baron RL. Benign and malignant portal vein thrombosis: differentiation by CT characteristics. AJR Am J Roentgenol. 1997;168:719-723. 
50. Song ZZ, Huang M, Jiang TA, et al. Diagnosis of portal vein thrombosis discontinued with liver tumors in patients with liver cirrhosis and tumors by contrast-enhanced US: a pilot study. Eur J Radiol. 2010;75:185-188.

51. Wallner B, Edelman RR, Finn JP, Mattle HP. Bright pleural effusion and ascites on gradient-echo MR images: a potential source of confusion in vascular MR studies. AJR Am J Roentgenol. 1990;155:1237-1240.

52. Senzolo M, Sartori M, Rossetto V, et al. Prospective evaluation of anticoagulation and transjugular intrahepatic portosystemic shunt for the management of portal vein thrombosis in cirrhosis. Liver Int. 2012;32:919-927.

53. De SA, Moscatelli R, Catalano C, et al. Systemic thrombolysis of portal vein thrombosis in cirrhotic patients: a pilot study. Dig Liver Dis. 2010;42:451-455.

54. Ravaioli M, Zanello M, Grazi GL, et al. Portal vein thrombosis and liver transplantation: evolution during 10 years of experience at the University of Bologna. Ann Surg. 2011;253:378-384.

55. Shi LW, Verran D, Chang D, et al. Primary liver transplantation with preexisting portal vein thrombosis. Transplant Proc. 2003;35: 354-355.

56. Doenecke A, Tsui TY, Zuelke C, et al. Pre-existent portal vein thrombosis in liver transplantation: influence of preoperative disease severity. Clin Transplant. 2010;24:48-55.

57. Bertelli R, Nardo B, Montalti R, et al. Liver transplantation in recipients with portal vein thrombosis: experience of a single transplant center. Transplant Proc. 2005;37:1119-1121.

58. Delgado MG, Seijo S, Yepes I, et al. Efficacy and safety of anticoagulation on patients with cirrhosis and portal vein thrombosis. Clin Gastroenterol Hepatol. 2012;10:776-783.

59. Huard G, Bilodeau M. Management of anticoagulation for portal vein thrombosis in individuals with cirrhosis: a systematic review. Int J Hepatol. 2012;2012:672986.

60. Amitrano L, Guardascione MA, Menchise A, et al. Safety and efficacy of anticoagulation therapy with low molecular weight heparin for portal vein thrombosis in patients with liver cirrhosis. J Clin Gastroenterol. 2010;44:448-451.

61. Bechmann LP, Sichau M, Wichert M, Gerken G, Kroger K, Hilgard P. Low-molecular-weight heparin in patients with advanced cirrhosis. Liver Int. 2011;31:75-82.

62. Tripodi A, Anstee QM, Sogaard KK, et al. Hypercoagulability in cirrhosis: causes and consequences. I Thromb Haemost. 2011;9:1715-1723.

63. Valla DC. Thrombosis and anticoagulation in liver disease. Hepatology. 2008;47:1384-1393.

64. Tripodi A, Mannucci PM. The coagulopathy of chronic liver disease. N Engl J Med. 2011;365:147-156.

65. Lisman T, Kamphuisen PW, Northup PG, Porte RJ. Established and new-generation antithrombotic drugs in patients with cirrhosis possibilities and caveats. J Hepatol. 2013;59:358-366.

66. Han G, Qi X, He C, et al. Transjugular intrahepatic portosystemic shunt for portal vein thrombosis with symptomatic portal hypertension in liver cirrhosis. J Hepatol. 2011;54:78-88.

67. Perarnau JM, Baju A, D'Alteroche L, Viguier J, Ayoub J. Feasibility and longterm evolution of TIPS in cirrhotic patients with portal thrombosis. Eur J Gastroenterol Hepatol. 2010;22:1093-1098.

68. Senzolo M, Burra P, Patch D, Burroughs AK. Tips for portal vein thrombosis in cirrhosis: not only unblocking a pipe. J Hepatol. 2011;55:945-946.

69. Senzolo M, Tibbals J, Cholongitas E, Triantos CK, Burroughs AK, Patch D. Transjugular intrahepatic portosystemic shunt for portal vein thrombosis with and without cavernous transformation. Aliment Pharmacol Ther. 2006;23:767-775.
70. Luca A, Miraglia R, Caruso S, et al. Short and long-term effects of the transjugular intrahepatic portosystemic shunt on portal vein thrombosis in patients with cirrhosis. Gut. 2011;60:846852.

71. Ferral H, Gamboa P, Postoak DW, et al. Survival after elective transjugular intrahepatic portosystemic shunt creation: prediction with model for end-stage liver disease score. Radiology. 2004; 231:231-236.

72. Hollingshead M, Burke CT, Mauro MA, Weeks SM, Dixon RG, Jaques PF. Transcatheter thrombolytic therapy for acute mesenteric and portal vein thrombosis. J Vasc Interv Radiol. 2005;16:651-661.

73. Villa E, Zecchini R, Marietta M, et al. Enoxaparin prevents portal vein thrombosis and decompensation in advanced cirrhotic patients: final report of a prospective randomized controlled study. Hepatology. 2011;54:418A-419A.

74. Nevens F, Bustami R, Scheys I, Lesaffre E, Fevery J. Variceal pressure is a factor predicting the risk of a first variceal bleeding: a prospective cohort study in cirrhotic patients. Hepatology. 1998;27: 15-19.

75. Tsochatzis EA, Senzolo M, Germani G, Gatt A, Burroughs AK. Systematic review: portal vein thrombosis in cirrhosis. Aliment Pharmacol Ther. 2010;31:366-374.

76. Garcia-Tsao G, Sanyal AJ, Grace ND, et al. AASLD practice guidelines: prevention and management of gastroesophageal varices and variceal hemorrhage in cirrhosis. Hepatology. 2007;46:922938.

77. Van Thiel DH, Schade RR, Starzl TE, et al. Liver transplantation in adults. Hepatology. 1982;2:637-640.

78. Shaw Jr BW, Iwatsuki S, Bron K, Starzl TE. Portal vein grafts in hepatic transplantation. Surg Gynecol Obstet. 1985;161:66-68.

79. Ponziani FR, Zocco MA, Campanale C, et al. Portal vein thrombosis: insight into physiopathology, diagnosis, and treatment. World J Gastroenterol. 2010;16:143-155.

80. Wu TH, Lin YS, Lee CF, et al. Clinical analysis and strategy for liver transplantation in patients with pre-existing portal vein thrombosis. Chang Gung Med J. 2011;34:426-434.

81. Rodriguez-Castro KI, Porte RJ, Nadal E, Germani G, Burra P, Senzolo M. Management of nonneoplastic portal vein thrombosis in the setting of liver transplantation: a systematic review. Transplantation. 2012;94:1145-1153.

82. Paskonis M, Jurgaitis J, Mehrabi A, et al. Surgical strategies for liver transplantation in the case of portal vein thrombosis - current role of cavoportal hemitransposition and renoportal anastomosis. Clin Transplant. 2006;20:551-562.

83. Lendoire J, Raffin G, Cejas N, et al. Liver transplantation in adult patients with portal vein thrombosis: risk factors, management and outcome. HPB (Oxford). 2007;9:352-356.

84. Gimeno FA, Calvo J, Loinaz C, et al. Comparative analysis of the results of orthotopic liver transplantation in patients with and without portal vein thrombosis. Transplant Proc. 2005;37:3899-3903.

85. Ceulemans B, Aerts R, Monbaliu D, et al. Liver transplantation using cavoportal transposition: an effective treatment in patients with complete splanchnic venous thrombosis. Transplant Proc. 2005; 37:1112.

86. Urbani L, Cioni R, Catalano G, et al. Cavoportal hemitransposition: patient selection criteria and outcome. Transplant Proc. 2002;34:3331.

87. Arcadipane A, Nadalin S, Gruttadauria S, et al. The recipient with portal thrombosis and/or previous surgery. Transplant Proc. 2008;40:1183-1186. 